\title{
Leitthema
}

Bundesgesundheitsbl 2016 $59: 584-591$

DOI 10.1007/s00103-016-2334-7

Online publiziert: 18. April 2016

๑) Springer-Verlag Berlin Heidelberg 2016

CrossMark

\author{
Nadine Zeitlmann ${ }^{1,2,3} \cdot$ Maja George $^{1,2,4} \cdot$ Gerhard Falkenhorst $^{1}$ \\ 'Abteilung für Infektionsepidemiologie, Robert Koch-Institut, Berlin, Deutschland \\ ${ }^{2}$ Postgraduiertenausbildung für angewandte Epidemiologie (PAE), Robert Koch-Institut, Berlin, \\ Deutschland \\ ${ }^{3}$ Sachbereich Infektionsepidemiologie, Bayerisches Landesamt für Gesundheit und \\ Lebensmittelsicherheit, Oberschleißheim, Deutschland \\ ${ }^{4}$ Infektionsepidemiologie, Landesuntersuchungsamt Rheinland-Pfalz, Landau, Deutschland
}

\section{Polioimpfung und Stuhlscreening in deutschen \\ Erstaufnahmeeinrichtungen für Asylsuchende, November 2013-Januar 2014}

\section{Was wurde umgesetzt?}

\section{Zusatzmaterial online}

Zusätzliche Informationen sind in der Online-Version dieses Artikels (DOI: 10.1007/ s00103-016-2334-7) enthalten.

Poliomyelitis (Polio) wird durch Polioviren verursacht. Die Übertragung erfolgt hauptsächlich auf fäkal-oralem Weg von Mensch zu Mensch [1]. Bei über $95 \%$ der Infizierten verläuft eine Infektion mit dem Poliovirus asymptomatisch oder mit unspezifischen Symptomen, sie können das Virus jedoch durch Stuhl ausscheiden. Bei circa einem von 200 Infizierten kommt es durch Befall des zentralen Nervensystems zur paralytischen Poliomyelitis, die sich durch akute schlaffe Lähmungen („Kinderlähmung“) auszeichnet [2,3]. Es gibt keine kausale Therapie gegen Polio [1]. Es existieren jedoch zwei Impfstoffarten: die PolioLebendimpfstoffe (orale Poliovakzine, OPV) und die inaktivierten Poliovakzine (IPV), die als Injektion geimpft werden [4, 5]. Die Grundimmunisierung gegen Polio sollte im 1. Lebensjahr erfolgen [6].

Polio war einst weltweit verbreitet [1]. Im Rahmen der 1988 begonnenen weltweiten Polioeradikationsinitiative [7] konnten der amerikanische, europäische, westpazifische sowie 2014 auch der südasiatische Raum für poliofrei erklärt werden [3]. In Deutschland trat die letzte autochthone Polioerkrankung im Jahre 1990 auf, und im Jahre 1992 wurden die letzten beiden importierten Fälle registriert [8]. Aktuell ist Polio noch in zwei Ländern ohne Unterbrechung endemisch: Pakistan und Afghanistan. In mehreren, vor allem afrikanischen und arabischen Ländern traten nach erfolgter Eradikation jedoch Ausbrüche durch importierte Polioviren oder vom Impfvirus abgeleitete Polioviren (sogenannte circulating vaccine-derived polioviruses, cVDPV) auf [9].

\section{Ausbruch von Poliomyelitis in Syrien}

Zwischen Juli 2013 und Januar 2014 wurden in einigen Provinzen Syriens gehäuft Fälle akuter schlaffer Lähmungen beobachtet, unter welchen bei 39 Fällen Polio-Wildvirus Typ 1 nachgewiesen werden konnte. Dies waren die ersten gemeldeten Poliofälle in Syrien seit 1999 [9]. Ein Großteil der Erkrankten waren nicht oder nicht vollständig geimpfte Kinder unter drei Jahren, wofür die aufgrund der Bürgerkriegssituation gesunkenen Polioimpfquoten in Syrien verantwortlich gemacht wurden. Diese waren nach Angaben des syrischen Gesundheitsministeriums von $99 \% \mathrm{im} \mathrm{Jahr}$ 2010 auf $68 \%$ im Jahr 2012 gesunken [10]. Für Deutschland wurde befürchtet, dass es angesichts der vielen Asylsuchenden aus Syrien zu einer Einschleppung von Polioviren kommen könnte. Die Zahl der Erstanträge von syrischen Asylsuchenden in Deutschland stieg von $6201 \mathrm{im}$ Jahre 2012 auf 11.851 im Jahre 2013 an $[11,12]$.

In Deutschland gibt es pro Bundesland eine je nach Asylaufkommen variierende Anzahl an Erstaufnahmeeinrichtungen (EAE) [13]. Die Verteilung der Asylsuchenden auf die EAE der Bundesländer erfolgt zentral durch das Bundesamt für Migration und Flüchtlinge (BAMF). Im Jahre 2013 wurden Asylsuchende mit dem Herkunftsland Syrien auf alle 16 Bundesländer verteilt [14]. 


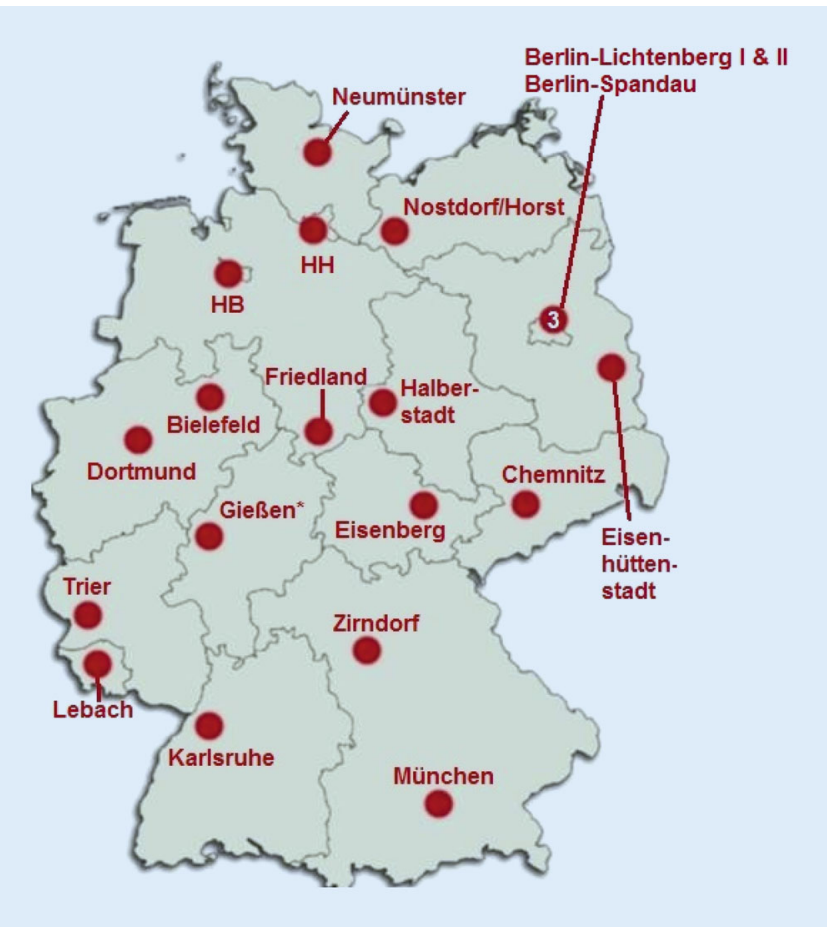

und darüber, welche Änderungen und Anpassungen bei bestehenden und zukünftigen Impf- und Screeningempfehlungen für Asylsuchende miteinbezogen werden sollten.

\section{Methoden}

Die Evaluation der Umsetzung von Impfempfehlung und Stuhlscreening auf Polioviren wurde in einer Umfrage mithilfe eines elektronischen Fragebogens durchgeführt. In einem ersten Schritt wurden die Infektionsschutzbeauftragten der Bundesländer per E-Mail gebeten, jede EAE in ihrem Bundesland sowie einen Ansprechpartner zu nennen, der in dieser EAE für das Thema Gesundheit von Asylsuchenden zuständig ist.

Für die genannten Ansprechpartner wurde ein Fragebogen im MicrosoftWord-Format entwickelt, im Testlauf an zwei EAE getestet und am 4.3.2014 per E-Mail an die identifizierten EAE ausgesandt. Er bezog sich auf den Zeitraum vom 1. November 2013 bis 31 . Januar 2014 und enthielt Fragen über allgemeine Angaben zur EAE, logistische Umsetzung der Impf- und Stuhlscreeningempfehlung, Zahlen $\mathrm{zu}$ in diesem Zeitraum angekommenen, geimpften und auf Poliovirenausscheidung untersuchten Asylsuchenden sowie Skalen zur Umsetzbarkeit und Akzeptanz der Empfehlung und ihren Informationsblättern (Fragebogen online als elektronisches Zusatzmaterial verfügbar).

Pro EAE wurden folgende Informationen ausgewertet:

- Personengruppen, denen eine Impfung angeboten wurde,

- die an der Impfung beteiligten Hauptakteure,

- Beginn der Impfungen,

- das Vorhandensein von Impfpässen bei Asylsuchenden,

- der Anteil der Asylsuchenden unter den Angekommenen ohne Impfpass, der im Rahmen der Empfehlung geimpft wurde (Impfquote, nach unten stehender Formel),

- der Anteil der auf Polioausscheidung getesteten Asylsuchenden unter den Angekommenen (StuhlscreeningQuote),
Hieraus sollten wichtige Erkenntnisse über die Durchführung von Impfungen in deutschen EAE gewonnen werden
2. Zusätzlich wurde ein temporäres Stuhlscreening auf Polioviren bei 
Bundesgesundheitsbl 2016 ·59:584-591 DOI 10.1007/s00103-016-2334-7

(c) Springer-Verlag Berlin Heidelberg 2016

\section{N. Zeitlmann · M. George · G. Falkenhorst}

\section{Polioimpfung und Stuhlscreening in deutschen Erstaufnahmeeinrichtungen für Asylsuchende, November 2013-Januar 2014. Was wurde umgesetzt?}

\section{Zusammenfassung}

Hintergrund. Aufgrund des Polio-Ausbruchs in Syrien und der steigenden Anzahl von Asylsuchenden aus Syrien in Deutschland 2013 empfahl das Robert Koch-Institut am 1.11.2013 im Rahmen der bestehenden Impfempfehlungen für Asylsuchende vordringlich die Polioimpfung syrischer Asylsuchender. Für syrische Asylsuchende unter drei Jahren wurde zusätzlich ein Stuhlscreening auf Poliovirusausscheidung empfohlen. Ziel. Dieser Beitrag evaluierte die Umsetzung beider Empfehlungen in den deutschen Erstaufnahmeeinrichtungen (EAE), um Erkenntnisse zur Durchführung von Impfungen in EAE gewinnen und zukünftige Empfehlungen anpassen zu können.
Methode. Alle deutschen EAE erhielten einen elektronischen Fragebogen, in dem für den Zeitraum vom 1.11.2013 bis 31.1.2014 um folgende Informationen gebeten wurde: allgemeine Angaben zur EAE und zur Durchführung von Impfungen, Haupthindernisse bei der Empfehlungsumsetzung, Zahlen zu in der EAE angekommenen, geimpften und auf Poliovirusausscheidung untersuchten Asylsuchenden sowie Umsetzbarkeit und Bewertung der Empfehlung und der begleitenden mehrsprachigen Informationsblätter. Ergebnisse und Diskussion. Alle 20 identifizierten EAE antworteten. Im Studienzeitraum kamen 33.874 Asylsuchende in den EAE an. Im Mittel besaßen, nach Angaben der EAE, $1,6 \%$ der Asylsuchenden einen Impfpass. Alle
EAE begannen zeitnah mit der Polioimpfung syrischer Asylsuchender unter drei Jahren; acht EAE erreichten hier Impfquoten von $\geq 80 \%$. Beim Stuhlscreening waren dies fünf von $19 \mathrm{EAE} .11 \mathrm{EAE}$ bewerteten beide Empfehlungen als "sehr gut/gut umsetzbar". Personalmangel und Sprachbarrieren, welche als Haupthindernisse der Umsetzung genannt wurden, könnten in zukünftigen Empfehlungen durch Zusammenarbeit mit Hilfsorganisationen und Einsatz von Informationsmaterial in weiteren Sprachen, wie fünf EAE vorschlugen, gemindert werden.

Schlüsselwörter

Polio $\cdot$ Impfung $\cdot$ Stuhlscreening $\cdot$ Asylsuchende $\cdot$ Deutschland

\section{Polio vaccination and stool screening in German reception centers for asylum seekers, November 2013-January 2014. What was implemented?}

\section{Abstract}

Background. Following the polio outbreak in Syria and the rising number of Syrian asylum seekers in Germany in 2013, the Robert Koch Institute recommended - within the context of existing vaccination recommendations for asylum seekers - on 01/11/2013 to prioritize polio vaccination of Syrian asylum seekers and stool screening in a target group of Syrian asylum seekers aged less than three years. Objectives. The article evaluates the implementation of this recommendation in German asylum seeker reception centres (RC) to gain further knowledge on the vaccination practices in RCs and to identify opportunities for improving future recommendations. Methods. The electronic questionnaire was sent by email to all German RCs, asking for general information on the RC, existing vaccination efforts, the main obstacles for implementation of the recommendations, the number of incoming and vaccinated asylum seekers, and asylum seekers screened for poliovirus in the period from $01 / 11 / 2013$ to $31 / 01 / 2014$. The $\mathrm{RCs}$ rated the feasibility of the recommendation and the provided multilingual information material.

Results and Conclusion. All of the 20 identified RCs responded. During the study period, 33.874 asylum seekers arrived in the RCs. Of those with available information about possession of a vaccination record, on average $1.6 \%$ did have one. All RCs offered timely vaccination to Syrian asylum seekers younger than three years. In this target group, eight
$\mathrm{RC}$ achieved vaccination coverages of $\geq 80 \%$. Stool screening coverage was $\geq 80 \%$ in five of $19 \mathrm{RCs}$. Eleven RCs rated the recommendation as very well/well implementable. Staff shortages and language barriers were mentioned as the main implementation obstacles. Similar future recommendations for asylum seekers in RCs should be accompanied by informational material in additional languages. Staff shortages hampering implementation could be overcome through collaborations with non-governmental organizations.

\section{Keywords}

Polio · Vaccination · Stool screening · Asylum seekers · Germany
- die zwei häufigsten Hindernisse bei der Umsetzung der Impfbeziehungsweise StuhlscreeningEmpfehlung,

- die selbst eingeschätzte Umsetzbarkeit und Akzeptanz der Empfehlungen,

- die Nützlichkeit der Informationsmaterialien zur Durchführung und

- die am häufigsten genannten Verbesserungsvorschläge.
Die Impfquote im Rahmen der Empfehlung wurde aus den angegebenen Anzahlen im Fragebogen nach folgender Formel berechnet:

$$
\begin{aligned}
& \text { Impfquote }= \\
& N(\text { geimpfte Asylsuchende }) / \\
& (N[\text { angekommene Asylsuchende }]- \\
& N[\text { Asylsuchende mit Impfpass }])
\end{aligned}
$$

Gaben EAE im Fragebogen für den Nenner Zahlen von angekommen syrischen Asylsuchenden an, die nicht altersstratifiziert (über und unter drei Jahre alt) vorlagen, wurde die gleiche Altersverteilung angenommen, die aus einem Datensatz des BAMF zu ankommenden syrischen Asylsuchenden in den Jahren 2013 und 2014 bekannt war [17].

In einem letzten Schritt wurde mit Ranksum-Tests und Fishers exakten Tests 


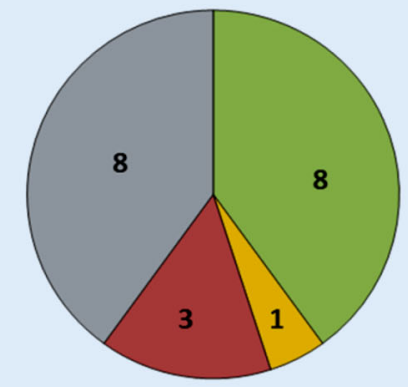

Syrische $A B<3$ Jahren (Impfangebot in 20 EAE)

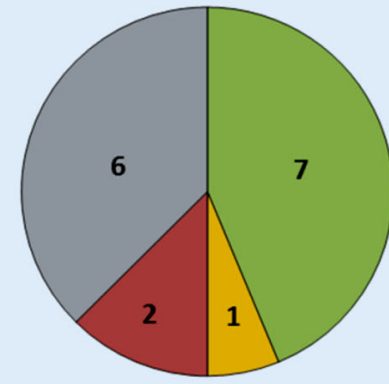

Syrische Asylsuchende aller Altersklassen (Impfangebot in 16 EAE)

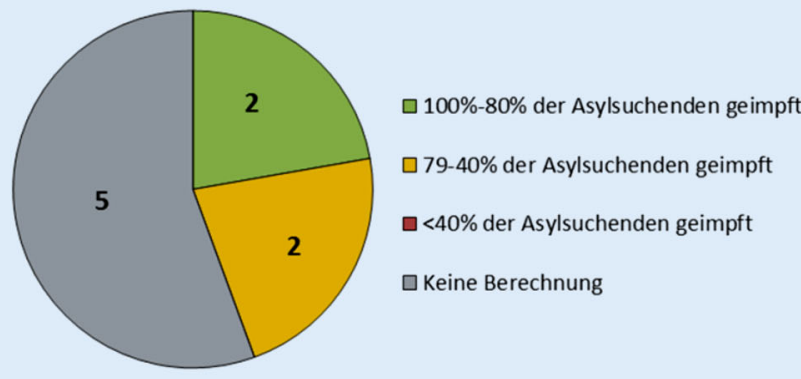

Asylsuchende anderer

Herkunftsländer

(Impfangebot in 9 EAE)

Abb. $2 \Delta$ Anzahl derErstaufnahmeeinrichtungen für Asylsuchende(EAE), die dieangegebenen Impfquoten in verschiedenen Gruppen von Asylsuchenden erreicht haben

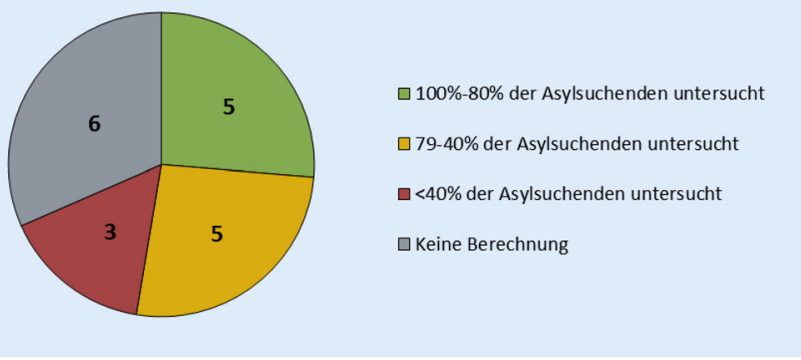

Abb. $3<$ Anzahl der Erstaufnahmeeinrichtungen für Asylsuchende (EAE), die die angegebenen Stuhlscreeningquoten bei Kindern unter 3 Jahren aus Syrien erreicht haben

geprüft, ob die berechneten Anteile geimpfter und auf Poliovirusausscheidung untersuchten Asylsuchenden sowie die Umsetzbarkeit der Empfehlungen von

- der durchschnittlichen Belegungszahl der EAE,

- der durchschnittlichen Aufenthaltsdauer von Asylsuchenden in der EAE,

- einem bereits vor der RKI-Empfehlung vom November 2013 bestehenden routinemäßigen Impfund Stuhlscreening-Angebot für Asylsuchende

abhingen.

Die Auswertung erfolgte mit dem Statistikprogramm STATA 12.0.

\section{Ergebnisse}

Es wurden 20 EAE identifiziert. Alle beteiligten sich an der Befragung (• Abb. 1). In den 20 EAE kamen im Zeitraum der Studie 33.874 Asylsuchende an: 236 Asylsuchende unter drei Jahren aus Syrien, 4604 ältere Asylsuchende aus Syrien und 29.034 Asylsuchende aus anderen Her- kunftsländern. Je nach EAE lag die tägliche Belegungszahl zwischen 150 und 1900 (Median: 468 Asylsuchende), die durchschnittliche Aufenthaltsdauer bewegte sich zwischen 2,5 und 330 Tagen (Median: 38 Tage).

Die Ansprechpartner für Gesundheit von Asylsuchenden in den jeweiligen EAE, die den Autoren genannt wurden, kamen je nach Bundesland aus verschiedenen Behörden. Für 12 der 20 EAE wurde ein Ansprechpartner aus dem Gesundheitsamt im Landkreis der EAE genannt, bei einer EAE kam der Ansprechpartner aus einer Nichtregierungsorganisation.

\section{Umsetzung der Impfempfehlung}

\section{Impfung von Asylsuchenden}

Für 10.387 (31\%) Asylsuchende wurde von den EAE angegeben, ob ein Impfpass vorlag; dieser lag bei 167 Asylsuchenden (1,6\%) vor. Bei syrischen Asylsuchenden unter drei Jahren hatten von den 153 (64\%) angekommenen Asylsuchenden, von welchen diese Information vorlag, 37 (25\%) einen Impfpass. Bei älteren Asyl- suchenden aus Syrien $(n=1879,41 \%)$ und Asylsuchenden anderer Herkunftsländer $(n=8355,29 \%)$ lagen diese Werte bei unter $7 \%$ beziehungsweise $1 \%$.

Zum Zeitpunkt der Evaluation boten fünf EAE Asylsuchenden generelle Routineimpfungen entsprechend der STIKOEmpfehlung an. In einer EAE geschah dies für alle von der STIKO empfohlenen Impfungen und für alle Asylsuchende ungeachtet des Herkunftslandes.

In allen 20 EAE wurde die Polioimpfung für syrische Asylsuchende unter drei Jahren angeboten. In 16 EAE wurde dies aufalle Asylsuchenden mit syrischer Herkunft ausgeweitet, und neun EAE schlossen alle Asylsuchende ungeachtet ihres Herkunftslandes in das Impfangebot im Rahmen der Empfehlung ein. Fünf EAE boten sowohl allen Asylsuchenden als auch dem Personal der EAE eine Polioimpfung an. Die Impfquoten wurden für die einzelnen Personengruppen stratifiziert und nur unter Einbeziehung der EAE berechnet, die ein Angebot für diese Personengruppen hatten. Bezüglich syrischer Kinder unter drei Jahren, für die in allen EAE ein Impfangebot bestand, erreichten acht EAE Impfquoten von $80 \%$ oder höher. Sieben der $16 \mathrm{EAE}$, die die Impfung auch älteren syrischen Asylsuchenden anboten, erreichten Quoten von $80 \%$ oder höher bei dieser Personengruppe. Von den neun EAE, die die Impfung auch Asylsuchenden anderer Herkunftsländer anboten, erreichten zwei EAE in dieser Personengrup- 
Tab. 1 Hindernisse bei der Umsetzung der RKI-Empfehlungen vom 1. November 2013 zur Polioimpfung und zum Stuhlscreening in den Erstaufnahmeeinrichtungen für Asylsuchende (EAE) (Jede EAE konnte maximal zwei Hindernisse pro Empfehlung angeben.)

\begin{tabular}{|lll|}
\hline $\begin{array}{l}\text { Am häufigsten genannte Hindernisse bei der } \\
\text { Umsetzung }\end{array}$ & $\begin{array}{l}\text { der Impfung } \\
\text { (Anzahl EAE) }\end{array}$ & $\begin{array}{l}\text { des Stuhlscreenings } \\
\text { (Anzahl EAE) }\end{array}$ \\
\hline Personalmangel & 11 & 5 \\
\hline Sprachbarrieren & 7 & 10 \\
\hline Undefinierbarer Impfstatus & 7 & - \\
\hline Ablehnung durch Asylsuchende & 6 & 3 \\
\hline Logistische Schwierigkeiten & 5 & 8 \\
\hline Finanzielle Engpässe/Kostenübernahme ungeklärt & 1 & 2 \\
\hline Keine Hindernisse & 3 & 5 \\
\hline
\end{tabular}

pe eine Impfquote von $80 \%$ oder höher (• Abb. 2).

Für 13 der 20 EAE war das Datum des Eingangs der Empfehlung bekannt. Sieben der 13 EAE fingen vor dem offiziellen Erhalt der Empfehlung, noch am selben Tag oder bis zu sieben Tage nach Erhalt der Empfehlung mit der Impfung an. Die restlichen sechs EAE benötigten zwischen acht Tagen und einem Monat, bevor die erste Impfung durchgeführt werden konnte.

In 14 EAE wurden Asylsuchende direkt in der Einrichtung geimpft, bei den restlichen sechs EAE wurde nur außerhalb der EAE geimpft. Bei diesen sechs EAE wurde eine kürzere mittlere Aufenthaltsdauer der Asylsuchenden beobachtet (30 Tage) als bei den EAE, die in der Einrichtung impften (68 Tage). In fünf EAE wurde von niedergelassenen Ärzten, in ebenfalls fünf EAE von Ärzten des Gesundheitsamts, in drei EAE durch einen Arzt der Einrichtung und in den restlichen sieben EAE teils durch Ärzte des Gesundheitsamts, teils durch Ärzte der Einrichtung geimpft.

In allen EAE wurde geimpften Asylsuchenden ohne Impfpass nach der Impfung ein persönlicher Impfpass ausgehändigt, aber nur sechs EAE führten zusätzlich ein Impfregister in Papierform (zwei EAE), elektronisch (drei EAE) oder beides (ein EAE).

Drei der 20 EAE gaben an, die Impfung ohne Hindernisse durchgeführt $\mathrm{zu}$ haben. Bei 11 EAE behinderten Personalmangel, bei sieben EAE Sprachbarrieren und die Schwierigkeit, den Impfstatus des Asylsuchenden zu definieren, die Umsetzung (• Tab. 1).

\section{Impfung des Personals}

Eine der 17 EAE, die Angaben zu dieser Frage machte, gab die Verantwortung der Sicherstellung des Polioimpfschutzes beim Personal an den arbeitsmedizinischen Dienst ab und machte keine weiteren Angaben zur Umsetzung. Fünf der übrigen 16 EAE kontrollierten selbst die Impfpässe der Mitarbeiter, während 11 EAE die Mitarbeiter lediglich darauf hinwiesen, ihren Impfschutz überprüfen zu lassen.

War nach diesen Kontrollen/Kontrollaufforderungen kein Impfschutz vorhanden, wurde Mitarbeitern in sechs EAE direkt in der Einrichtung eine Impfung angeboten. In weiteren sechs EAE wurden die Mitarbeiter aufgefordert, sich bei ihrem Hausarzt impfen zu lassen. Es liegen keine Informationen vor, wie viele der Mitarbeiter diesem Impfangebot/der Impfaufforderung nachkamen.

\section{Umsetzung des Stuhlscreenings}

19 der 20 EAE beteiligten sich am Stuhlscreening auf Poliovirusausscheidung bei syrischen Asylsuchenden unter drei Jahren. Von diesen schloss eine EAE zusätzlich erwachsene Syrer in das Stuhlscreening mit ein, vier EAE untersuchten nur Kinder, weiteten die Empfehlung jedoch auf andere Herkunftsländer wie z. B. Afghanistan, Pakistan, Somalia und das Horn von Afrika aus. Eine der 19 EAE schickte Stuhlproben aller Asylsuchenden aller Altersklassen sowie auch des Personals der EAE zur Testung ein.

Stuhlscreeningquoten wurden nur für die Zielgruppe der unter 3-jährigen Asylsuchenden aus Syrien berechnet. Von 19 EAE, die sich am Stuhlscreening beteiligten, fehlten bei sechs EAE die nötigen Angaben zur Berechnung einer Stuhlscreeningquote, und fünf EAE untersuchten über $80 \%$ der angekommenen Asylsuchenden auf Polioausscheidung (davon vier EAE $100 \%$ ) (• Abb. 3).

Fünf EAE gaben an, dass sie die Stuhlscreeningempfehlung ohne Hindernisse umsetzen konnten. Ähnlich wie bei der Impfempfehlung berichteten 10 EAE, dass besonders Sprachbarrieren die Umsetzung behinderten. Logistische Schwierigkeiten wurden als zweithäufigstes Hindernis genannt (• Tab. 1).

Sieben EAE boten routinemäßiges Stuhlscreening auf die Ausscheidung anderer Darmpathogene (z. B. Salmonellen, Shigellen und Parasiten) schon vor der Empfehlung des RKI zum Screening auf Polioviren an. Unter diesen EAE mit etabliertem Routinescreening berichteten nur halb so viele ( $43 \%$ ) von einer Durchführung mit Hindernissen wie unter den 13 restlichen EAE ohne routinemäßiges Stuhlscreening (92\%) (p-Wert: 0,015).

\section{Beurteilung der RKI-Empfehlungen}

Insgesamt wurde die Empfehlung von 16 EAE als sehr sinnvoll oder sinnvoll angesehen, während zwei EAE sie für weniger oder überhaupt nicht sinnvoll hielten. Zwei EAE gaben keine Beurteilung ab. Auf einer Skala von „sehr gut“ bis „überhaupt nicht gut“ umsetzbar, klassifizierten 11 EAE die Empfehlung als „sehr gut oder gut umsetzbar“, während knapp die Hälfte der EAE $(n=9)$ sie als ,nicht gut umsetzbar", einstuften. Unter den großen EAE mit täglichen Belegungszahlen von über 600 Asylsuchenden fand sich die Einstufung einer geringeren Umsetzbarkeit der Empfehlung häufiger (63\%) als unter kleinen EAE (27\%).

Die Informationsblätter, die von RKI und der Bundeszentrale für gesundheitliche Aufklärung (BZgA) zur Verfügung gestellt worden waren, zeigten hohen $\mathrm{Be}$ kanntheitsgrad (17 bis 19 EAE, je nach Informationsblatt, $\bullet$ Tab. 2) und erhielten von fast allen EAE sehr gute bis gute Bewertungen (zwischen 17 und 18 EAE, abhängig vom Informationsblatt, ๑ Tab. 2).

Für zukünftige Empfehlungen schlugen fünf EAE vor, die Impfinformations- 


\begin{tabular}{|c|c|c|}
\hline Informationsblatt & Bekanntheit/Bewertung des Materials & $\begin{array}{l}\text { Anzahl EAE } \\
(N=20)\end{array}$ \\
\hline \multirow[t]{4}{*}{ Merkblatt Polio des RKI } & Material nicht bekannt & 1 \\
\hline & Material bekannt & 19 \\
\hline & - Bewertung sehr gut/gut & 17 \\
\hline & - Bewertung weniger/überhaupt nicht gut & 2 \\
\hline \multirow{4}{*}{$\begin{array}{l}\text { Impfaufklärungs- } \\
\text { materialien des RKI }\end{array}$} & Material nicht bekannt & 1 \\
\hline & Material bekannt & 19 \\
\hline & - Bewertung sehr gut/gut & 18 \\
\hline & - Bewertung weniger/überhaupt nicht gut & 1 \\
\hline \multirow{4}{*}{$\begin{array}{l}\text { Hygienemerkblatt der } \\
\text { Bundeszentrale für } \\
\text { gesundheitliche } \\
\text { Aufklärung (BZgA) }\end{array}$} & Material nicht bekannt & 3 \\
\hline & Material bekannt & 17 \\
\hline & - Bewertung sehr gut/gut & 17 \\
\hline & - Bewertung weniger/überhaupt nicht gut & 0 \\
\hline
\end{tabular}

blätter des RKI in zusätzlichen Sprachen zur Verfügung zu stellen.

\section{Diskussion}

Das Ziel dieser Evaluation wares, die Umsetzung der Empfehlungen des RKI vom 1. November 2013 zur Polioimpfung und zum Stuhlscreening bei Asylsuchenden zu quantifizieren sowie Hindernisse und begünstigende Faktoren für die Umsetzung der Empfehlung zu identifizieren.

\section{Impfempfehlung}

Zum Zeitpunkt der Veröffentlichung der Empfehlung folgten nur wenige EAE den allgemeinen STIKO-Empfehlungen für die routinemäßige Impfung von ankommenden Asylsuchenden. Im Rahmen der Empfehlung zur Polioimpfung gelang allen EAE jedoch ein zeitnaher Impfbeginn. Die RKI-Empfehlung wurde am besten für die Gruppe der syrischen Kinder unter drei Jahren umgesetzt.

Aus welchen Gründen für diese Zielgruppe nur acht EAE hohe Impfquoten ( $\geq 80 \%$ ) erreichten, wurde im Rahmen unserer Befragung nicht im Detail ermittelt. Zwei EAE gaben allerdings im Freitext an, dass auf eine Impfung verzichtet wurde, wenn ein vollständiger Polioimpfstatus durch mündliche Anamnese ermittelt worden war. Da diese Information nicht systematisch erfasst wurde, wurde mit der Impfquotenberechnung wahr- scheinlich der Anteil geschützter Personen unterschätzt.

Die Polioimpfquote unter Asylsuchenden mit vorhandenem Impfpass konnte nicht ermittelt werden, da im Rahmen dieser Evaluierung nicht erhoben werden konnte, ob diese Personen laut Impfpass vollständig gegen Polio geimpft waren oder ob sie weitere Polioimpfdosen benötigten. Impfregister wurden nur von rund jeder dritten EAE in Deutschland geführt, was die Ermittlung von Impfquoten bei Asylsuchenden erschwert.

In allen Einrichtungen wurde ungeimpftes Personal zur Impfung in der EAE oder beim Hausarzt aufgefordert. Inwieweit die Mitarbeiter der Aufforderung nachkamen, sich impfen zu lassen, wurde in dieser Evaluation aus erhebungstechnischen Gründen und Gründen des Datenschutzes nicht erfasst.

\section{Stuhlscreening}

Alle bis auf eine EAE untersuchten Stuhlproben der ankommenden Asylsuchenden auf Polioviren, wobei nur rund ein Drittel dieser EAE hohe Screeningquoten ( $\geq 80 \%$ ) erzielte.

Sechs EAE führten zum Zeitpunkt der Evaluation bereits ein routinemäßiges Stuhlscreening von Asylsuchenden auf andere Erreger durch. Diese EAE gaben auch an, dass die Umsetzung des Stuhlscreenings für sie ohne größere Schwierigkeiten möglich war. Ein bereits vor der Empfehlung routinemäßig durchgeführtes Stuhlscreening begünstigte daher die Umsetzung der Stuhlscreeningempfehlung.

In der gesamten Zeit des Stuhlscreenings (1. November 2013 bis 18. April 2014) wurden 425 Stuhlproben (inklusive Proben der Kontingentflüchtlinge) von Kindern unter drei Jahren aus Syrien untersucht. Die Stuhlproben wurden außer von EAE auch von anderen Gemeinschaftsunterkünften für Asylsuchende eingeschickt. Es wurde innerhalb der sieben Monate, in denen das Stuhlscreening stattfand, kein Polio-Wildtypvirus nachgewiesen [18]. Dies zeigt, dass das Risiko einer Einschleppung von Polio-Wildvirus nach Deutschland aus Syrien sehr gering war.

\section{Hindernisse bei der Umsetzung der Empfehlungen}

Hinsichtlich der Umsetzung der Empfehlungen zeigten sich deutliche Unterschiede zwischen den EAE. Je höher die Belegungszahl einer EAE war, desto schwieriger war es, die Empfehlungen umzusetzen. Als größte Probleme bei der Umsetzung der Empfehlungen wurden Personalmangel und Sprachbarrieren genannt. Zusätzlich ergaben sich durch die teilweise sehr rasche Verlegung der Asylsuchenden innerhalb weniger Tage aus den EAE in kleinere Gemeinschaftsunterkünftelogistische Probleme, insbesondere hinsichtlich der Abgabe der Stuhlproben.

Trotz der eindeutigen Empfehlung der STIKO, dass Personen ohne Impfdokumente als nicht geimpft angesehen und empfohlene Impfungen durchgeführt werden sollen, gab ein großer Teil der EAE unklaren Impfstatus als Haupthindernis zur Umsetzung der Impfempfehlung an. Hier sind offensichtlich weitere Schulungen erforderlich.

\section{Limitation der Berechnungen}

Die Impf- und Stuhlscreeningquoten in dieser Evaluation wurden auf Basis aggregierter Daten berechnet. Insbesondere im Hinblick auf den Nenner, das heißt, die Zahl der eingetroffenen Asylsuchenden, sind die Quellen und die Zuverlässig- 
keit der von den EAE gemachten Angaben unklar. Dies liegt zumindest teilweise an der Aufsplitterung der Zuständigkeiten für den allgemeinen Betrieb der EAE und die Erstuntersuchung der Asylsuchenden zwischen unterschiedlichen Behörden. Je nach Zielgruppe der Impfempfehlung konnten bei 30 bis $60 \%$ der EAE wegen unzureichender Angaben gar keine Impfquoten berechnet werden. Bei der Stuhlscreeningempfehlung war dies bei jeder dritten EAE der Fall.

\section{Empfehlungen}

Zur besseren Umsetzung zukünftiger Empfehlungen werden folgende Anpassungen vorgeschlagen:

1. Das RKI kann zusammen mit seinen Partnern Sprachbarrieren mindern, indem es weiteres mehrsprachiges Informationsmaterial zu Impfungen für die EAE zur Verfügung stellt. In der Zeit zwischen der Durchführung dieser Evaluation und Abfassung dieses Berichts wurde diese Empfehlung bereits weitgehend umgesetzt, und es stehen aktuell Informationsmaterialien zu verschiedenen Impfungen in 19 Fremdsprachen auf der RKI-Webseite zum Download zur Verfügung (Stand Februar 2016) [19].

2. Die zuständigen Akteure in Ministerien und Regierungen sollten auf möglicherweise auftretende personelle Engpässe bei der Umsetzung von Impf- und Screening-Empfehlungen vorbereitet sein und reagieren. Die im Jahre 2015 stark angestiegenen Zahlen an Asylsuchenden [20] verstärken dieses Problem nicht nur für vorübergehende Empfehlungen, sondern auch für die generelle Durchführung von Routineimpfungen für Asylsuchende.

Es sollte diskutiert werden, wie in dieser Situation - ähnlich wie in internationalen Notfallsituationen kurzzeitig zusätzliches Personal rekrutiert werden kann, zum Beispiel durch Zusammenarbeit mit Nichtregierungsorganisationen oder medizinischen Hilfsorganisationen.

3. Die bestehenden STIKO-Impfempfehlungen für Flüchtlinge und Asylsuchende in Gemeinschafts- unterkünften sollten in EAE und Gemeinschaftsunterkünften für Asylsuchende, ungeachtet von internationalen Ausbruchsgeschehen, routinemäßig umgesetzt werden.

4. Das RKI sollte bekräftigen, dass Asylsuchende mit unklarem Impfstatus als nicht geimpft anzusehen sind und aktuell empfohlene Impfungen auch bei fehlender Dokumentation früherer Impfungen erhalten können und sollen.

Durch diese Anpassungen kann nicht nur die Umsetzung zukünftiger Empfehlungen verbessert werden, sondern es kann auch die gesetzlich vorgeschriebene frühzeitige Gesundheitsversorgung von Asylsuchenden einschließlich der raschen Erkennung und Bekämpfung von Infektionsrisiken in Gemeinschaftsunterkünften gewährleistet werden. Auch zukünftige Empfehlungen sollten kurzfristig evaluiert werden, um Hindernisse bei der Umsetzung $\mathrm{zu}$ identifizieren und gegebenenfalls Anpassungen vornehmen zu können.

\section{Korrespondenzadresse}

\section{MPH N. Zeitlmann}

Abteilung für Infektionsepidemiologie, Robert Koch-Institut

Seestraße 10, 13353 Berlin, Deutschland ZeitlmannN@rki.de

Danksagung. Den Koordinatoren der Postgraduierten-Ausbildung für angewandte Epidemiologie (PAE) an den

Landesgesundheitsämtern in Bayern (Wolfgang Hautmann) und Rheinland-Pfalz (Florian Burckhardt), sowie der PAE-Koordinatorin Katharina Alpers am Robert Koch-Institut für ihre Unterstützung bei Planung, Umsetzung und Kommunikation der Ergebnisse. Katrin Neubauer von der Geschäftsstelle der Nationalen Kommission für die Polio-Eradikation in Deutschland für die Bereitstellung der Ergebnisse des Stuhlscreenings. Den teilnehmenden Ansprechpartnern für Gesundheit von Asylsuchenden der EAE in Deutschland.

\section{Einhaltung ethischer Richtlinien}

Interessenkonflikt. N. ZeitImann, M. George und G. Falkenhorst geben an, dass kein Interessenkonflikt besteht.

Dieser Beitrag beinhaltet keine von den Autoren durchgeführten Studien an Menschen oder Tieren.

\section{Literatur}

1. Heymann DL (2008) Acute poliomyelitis. In: American Public Health Association (Hrsg) Control of communicable diseases manual, 19. Aufl. American Public Health Association, Washington DC, S484-491

2. Wallace G, Alexander J (2015) Poliomyelitis. In: Centers for Disease Control (Hrsg) The pink book. epidemiology and prevention of vaccine preventable diseases, 13. Aufl. The Public Health Foundation, Atlanta, S297-310

3. WHO (2014) Poliomyelitis. fact sheet no 114. http:// www.who.int/mediacentre/factsheets/fs114/en/ (Erstellt:September 2015)

4. Salk JE, Krech U, Youngner JS et al (1954) Formaldehyd treatment and safety testing of experimental poliomyelitis vaccines. Am J Public Health Nations Health 44(5):563-570

5. Sabin $A B$ (1959) Present position of immunization against poliomyelitis with live virus vaccines. $\mathrm{Br}$ Med J 1 (5123):663-680

6. Robert Koch-Institut (2015) Empfehlungen der ständigen Impfkommission (STIKO) am Robert Koch-Institut/Stand: August 2015. Epidemiol Bull 34:327-362 (www.rki.de/DE/Content/Infekt/ EpidBull/Archiv/2015/Ausgaben/34_15.pdf? blob=publicationFile. Zugegriffen: 15 . September 2015) doi:10.17886/EPIBULL-2015-001

7. WHO (1988) Global eradication of poliomyelitis by the year 2000: resolution of the 41st world health assembly. www.who.int/ ihr/polioresolution4128en.pdf. Zugegriffen: 15. September 2015

8. Robert Koch-Institut (2013) Poliomyelitis. Ratgeber für Ärzte. www.rki.de/DE/Content/Infekt/ EpidBull/Merkblaetter/Ratgeber_Poliomyelitis. html. Zugegriffen: 15 . September 2015

9. (2014)The Global PolioEradication Initiative. www. polioeradication.org. Zugegriffen: 15 . September 2015

10. WHO (2015) Vaccine-preventable diseases: monitoring system. 2015 global summary. http://apps.who.int/immunization_monitoring/ globalsummary/countries?countrycriteria [country][]=SYR. Zugegriffen: 15. September 2015

11. Bundesamt für Migration und Flüchtlinge (2014) Anzahl der Asylsuchende im Jahr 2013: Höchster Stand seit 14 Jahren. www.bamf.de/SharedDocs/ Pressemitteilungen/DE/2014/20140110-0001pressemitteilung-bmi-asylzahlen-dezember. html. Zugegriffen: 10. Juli 2014

12. Bundesamt für Migration und Flüchtlinge (2013) Aktuelle Zahlen zu Asyl. Dezember 2013. http://www.bamf.de/SharedDocs/Anlagen/ EN/Downloads/Infothek/Statistik/statistikanlage-teil-4-aktuelle-zahlen-zu-asyl.pdf? blob=publicationFile (Erstellt: ). Zugegriffen: 15 . September 2015

13. Schneider J (2012) Working Paper 25. Die Organisation der Asyl- und Zuwanderungspolitik in Deutschland. Studie der deutschen nationalen Kontaktstelle für das Europäische Migrationsnetzwerks (EMN). http://ec.europa.eu/dgs/ home-affairs/what-we-do/networks/european $\% 5$ fmigration $\% 5$ fnetwork/reports/docs/emnstudies/migration-policies/10b.\%5fgermany $\% 5$ fnational $\% 5$ freport $\% 5$ forganisation $\% 5$ fof $\% 5$ fasylum $\% 5$ fand $\% 5$ fmigration $\% 5$ fpolicies \%5fde.pdf.Zugegriffen: 15. September 2015

14. Bundesamt für Migration und Flüchtlinge (2014) Antragstellung. www.bamf.de/DE/Migration/ AsylFluechtlinge/Asylverfahren/Antragstellung/ 
antragstellung-node.html. Zugegriffen: 10. Juli 2014

15. Robert Koch-Institut (2013) Empfehlungen der ständigen Impfkommission (STIKO) am Robert Koch-Institut/Stand: August 2013. Epidemiol Bull 34:340 (www.rki.de/DE/Content/Infekt/ EpidBull/Archiv/2013/Ausgaben/34_13.pdf? blob=publicationFile. Zugegriffen: 15 . September 2015)

16. Robert Koch-Institut (2014) Poliomyelitis-Ausbruch in Syrien: Beendigung der Stuhl-Surveillance bei Asylbewerbern. Empfehlung des RobertKoch Instituts. http://www.rki.de/DE/Content/ InfAZ/P/Polio/Ausbruch Syrien/Polio Syrien Empfehlung_Beendigung_Surveillance.pdf?_blob=publicationFile. Zugegriffen: 13. April 2016

17. Bundesamt für Migration und Flüchtlinge (2014) Aufhältige syrische Staatsangehörige mit Asylantragstellung ab 15. Oktober 2013 bis 30. Juni 2014. Datenbankauszug

18. Neubauer K, Boettcher S, Baillot A et al (2014) Stool screening for wild polio viruses (WPV) among syrian refugees and asylum seekers, germany, 2013/2014. european scientific conference on applied infectious disease epidemiology. http://ecdc. europa.eu/en/ESCAIDE/programme/abstractbook/Documents/ECSAIDE-2014-abstracts.PDF. Zugegriffen: 15. September 2015

19. Robert Koch-Institut (2015) Informationsmaterialien in verschiedenen Sprache. http://www. rki.de/DE/Content/Infekt/Impfen/Materialien/ materialien_fremdsprachig_inhalt.html. Zugegriffen: 12. Februar 2016

20. Bundesamt für Migration und Flüchtlinge (2015) Aktuelle Zahlen zu Asyl. August 2015. www.bamf.de/SharedDocs/Anlagen/ EN/Downloads/Infothek/Statistik/statistikanlage-teil-4-aktuelle-zahlen-zu-asyl.pdf? blob=publicationFile. Zugegriffen: 15 . September 2015 